
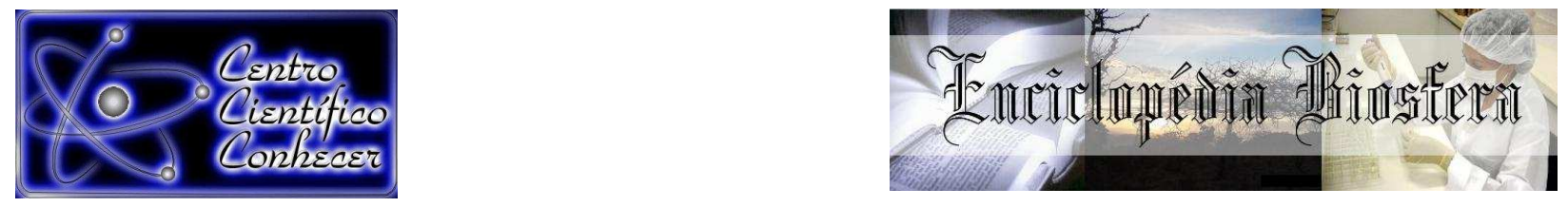

\title{
PRODUTIVIDADE DO COENTRO VERDÃO EM FUNÇÃO DAS REGULAGENS DE UMA SEMEADORA MANUAL
}

\author{
Josefa Maria Francieli da Silva ${ }^{1}$, Antônio Alves Pinto ${ }^{2}$, Laudeline Dantas Santana ${ }^{2}$, \\ Wíctor Állyson Dias Rodrigues ${ }^{2}$, Felipe Thomaz da Camara ${ }^{3}$ \\ ${ }^{1}$ Mestranda do Programa de Agronomia/Fitotecnia da Universidade Federal do \\ Ceará, Fortaleza-Brasil (francielisilva39@yahoo.com.br) \\ ${ }^{2}$ Discente do Curso de Agronomia da Universidade Federal do Cariri - UFCA, Crato, \\ Ceará, Brasil \\ ${ }^{3}$ Professor Doutor do curso de Agronomia da Universidade Federal do Cariri - \\ UFCA, Crato, Ceará, Brasil
}

Recebido em: 03/10/2016 - Aprovado em: 21/11/2016 - Publicado em: 05/12/2016 DOI: 10.18677/EnciBio_2016B 080

\begin{abstract}
O objetivo com este trabalho foi avaliar a produtividade do coentro verdão em função das regulagens de uma semeadora manual. $\mathrm{O}$ estudo foi conduzido no Centro de Ciências Agrárias e da Biodiversidade, da Universidade Federal do Cariri, Crato-CE, localizada na região do Cariri Cearense. $O$ delineamento usado foi em blocos casualizados, em esquema fatorial $2 \times 3$, com três repetições, totalizando 18 observações. O primeiro fator foram dois modelos de discos horizontais (Fileira dupla e fileira simples de furos redondos); e o segundo o número de dentes da engrenagem movida (15; 24 e 32 dentes). Cada parcela experimental foi composta por quatro fileiras espaçadas a $0,3 \mathrm{~m}$ com quatro metros de comprimento, totalizando uma área de $4,8 \mathrm{~m}^{2}$, sendo a parcela útil composta pelas duas fileiras centrais com dois metros de comprimento $\left(1,2 \mathrm{~m}^{2}\right)$. As variáveis analisadas foram à altura de plantas $(\mathrm{cm})$, número de plantas por metro na fileira e por metro quadrado, a massa por planta (g), a massa de plantas por metro de fileira (g) e a produtividade de coentro $\left(\mathrm{g} \mathrm{m}^{-2}\right)$. A Massa de planta por metro e a produtividade foram estatisticamente significativas tanto com relação ao modelo de disco quanto ao número de dentes, sendo que o disco com fileira dupla e com 15 dentes na engrenagem movida demostraram os melhores resultados. Conclui-se que a maior produtividade e número de plantas do coentro verdão foi com o disco de fileira dupla e para a engrenagem movida com 15 dentes.
\end{abstract}

RESUMO

PALAVRAS-CHAVE: coriandrum sativum, mecanização, população

\section{PRODUCTIVITY VERDÃO CORIANDER DEPENDING ON THE SETTINGS OF A MANUAL SEEDER}

\begin{abstract}
The objective of this research was to evaluate the productivity of verdão coriander depending on the settings of a manual seeder. The study was conducted at the Centre for Agricultural Sciences and Biodiversity, the Federal University of Cariri, Crato-CE, located in the Cariri Cearense region. The experimental design used was randomized blocks, in $2 \times 3$ factorial design with three replications, totaling 18
\end{abstract}


observations. The first factor were two models of horizontal discs (double row and single row of round holes); and according to the number of teeth of the driven gear (15, 24 and 32 teeth). Each plot consisted of four rows spaced at $0.3 \mathrm{~m}$ with four meters long, with a total area of $4.8 \mathrm{~m}^{-2}$ and the useful portion composed of the two central rows with two meters long $\left(1.2 \mathrm{~m}^{-2}\right)$. The variables analyzed were the plant height $(\mathrm{cm})$, number of plants per meter in the row and per square meter, the mass per plant $(\mathrm{g})$, the mass of plants per row meter $(\mathrm{g})$ and coriander productivity $\left(\mathrm{g} \mathrm{m}^{-2}\right)$. The plant mass per meter and productivity were statistically significant both in relation to the disc model and the number of teeth, and the double row disc 15 and the driven gear teeth have demonstrated the best results. It follows that the greater productivity and number of plants was verdão coriander with double row disc and the driven gear with 15 teeth.

KEYWORDS: coriandrum sativum, mechanization, population

\section{INTRODUÇÃO}

Tendo em vista a conscientização da população por uma dieta alimentar rica e saudável, o consumo de hortaliças tem aumentado sensivelmente (TAVELLA, 2010). O coentro (Coriandrum sativum) é uma hortaliça herbácea anual pertencente à família Apiaceae, popularmente utilizado na culinária nordestina, cujas folhas são utilizadas na composição e decoração de diversos pratos regionais. Embora, seja considerada uma "cultura de fundo de quintal", grande número de produtores estão envolvidos com a sua exploração durante todo o ano, o que justifica sua importância social e econômica (GRANJEIRO, et al., 2011; LINHARES et al., 2015; RESENDE, et., 2015).

Entre as cultivares de coentro disponíveis no mercado, a cultivar Verdão é considerada líder em todo o Brasil, além de ser de ciclo precoce, em torno de 30 a 40 dias para a produção de folhas, dependendo da época do ano e da região. Esta cultivar é bastante vigorosa, com folhas de coloração verde-escura, excelente rusticidade e boa resistência às pragas e doenças (SOUSA et al., 2011; SILVA et al., 2012; HORTIVALE, 2015; ANGELI et al., 2016).

Dentre as mais distintas atividades na agricultura, que empregam o uso de máquinas, tem-se a semeadura, que representa uma das mais importantes etapas da produção agrícola pelo fato de propiciar o estabelecimento da cultura. Semear é uma técnica milenar e, de sua qualidade, dependem o sucesso e a produtividade de uma cultura agrícola (FRANCETTO et al., 2015).

No Brasil o cultivo do coentro ainda é realizado, preferencialmente por semeadura direta manual e em canteiros, de forma orgânica, utilizando mão de obra familiar, pois são poucas as pesquisas realizadas com o coentro, que incluam tecnologia adequada no sistema de produção, principalmente no tocante a mecanização agrícola, no que se refere ao desempenho da semeadora, relacionado à abertura do sulco e a deposição da semente em profundidade correta e em contato com o solo (MACIEL, et al., 2012).

Segundo MELO et al., (2013) a utilização de máquinas e equipamentos na agricultura de pequeno porte, proporciona melhorias na capacidade operacional, facilidade no trabalho do homem, melhorando assim a eficiência produtiva. $O$ objetivo, com este trabalho, foi avaliar a produtividade do coentro verdão em função das regulagens de uma semeadora manual. 


\section{MATERIAL E MÉTODOS}

O estudo foi conduzido no Centro de Ciências Agrárias e da Biodiversidade, da Universidade Federal do Cariri, Crato-CE, localizada na região do Cariri Cearense, situando-se a 442 m de altitude, com latitude sul de $7^{\circ}$ 14' $3,4^{\prime \prime}$ e longitude oeste de 39 22' 7,6", em um solo classificado como Argissolo Vermelho Amarelo, conforme classificação do mapa de solos da EMBRAPA (2013), de relevo suave ondulado e textura da camada superficial do solo classificada como francoarenosa.

O clima da região de acordo com a classificação de KÖPPEN (1948), é o BSw'h', caracterizado como semiárido, com temperaturas elevadas, as quais variam, anualmente, entre 24 a $26^{\circ} \mathrm{C}$ e, nos meses mais quentes, entre 26 a $30^{\circ} \mathrm{C}$. A precipitação média anual da região é de 900 a 1000 mm, estando distribuída em uma estação chuvosa que vai de dezembro a maio.

O delineamento usado foi em blocos casualizados, em esquema fatorial $2 \times 3$, com três repetições, totalizando 18 observações. Os primeiros fatores foram dois modelos de discos horizontais (Fileira dupla e fileira simples de furos redondos); e o segundo o número de dentes da engrenagem movida (15; 24 e 32 dentes). Cada parcela experimental foi composta por quatro fileiras espaçadas a $0,3 \mathrm{~m}$ com quatro metros de comprimento, totalizando uma área de $4,8 \mathrm{~m}^{2}$, sendo a parcela útil composta pelas duas fileiras centrais com dois metros de comprimento $\left(1,2 \mathrm{~m}^{2}\right)$.

Foi utilizada uma semeadora manual da Knapik, para a semeadura do coentro cultivar Verdão, com sistema de abertura de sulcos por discos duplos defasados, e o sistema de distribuição de sementes por discos horizontais (Figura 1), muito útil para pequenas áreas agrícolas e para uso em sistema de preparo do solo convencional (Aração e gradagem) em virtude de não possuir discos de corte de palhada, inviabilizando seu uso para o sistema plantio direto.

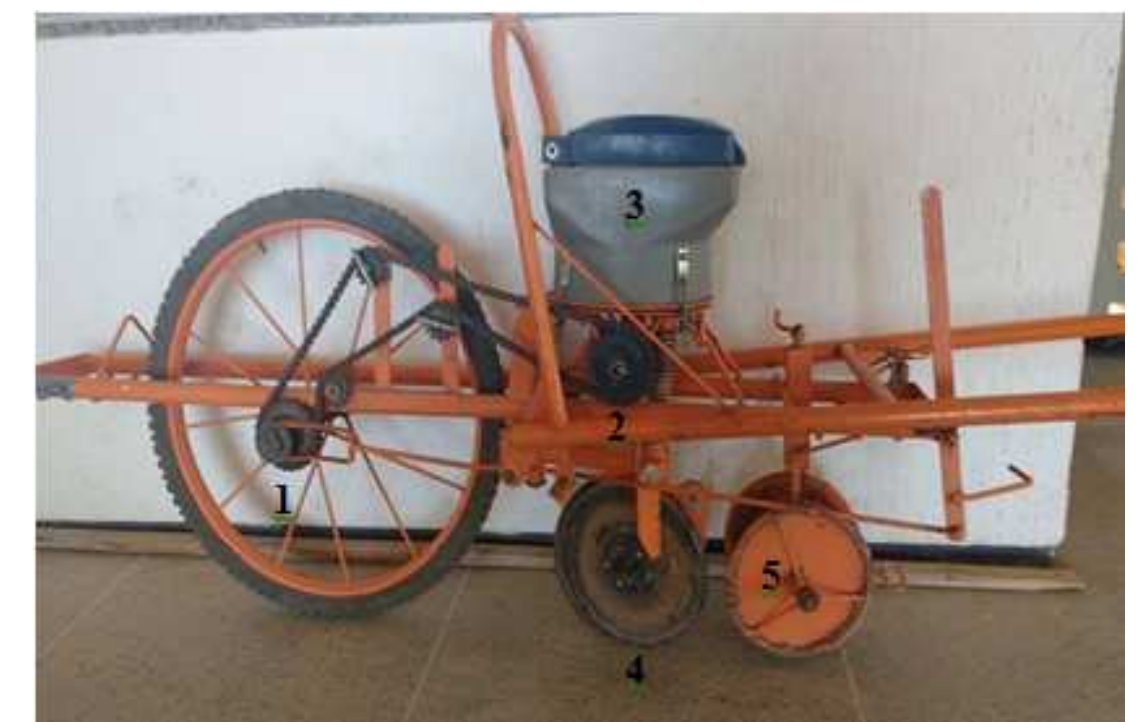

FIGURA 1 - Semeadora manual Knapik, com sistema de distribuição de sementes por discos horizontais, UFCA, Crato, 2016. (1 - Engrenagem motora; 2 Engrenagem movida; 3 - Depósito de sementes; 4 - Disco duplo defasado para abertura do sulco das sementes; 5 - Roda de controle de profundidade e cobertura dos sulcos).

Fonte: Autores 
Os discos horizontais utilizados foram os de formato redondo com fileira dupla (Figura 2A) e simples (Figura 2B), que são localizados no fundo do depósito de sementes e são responsáveis pela padronização na distribuição em função da relação de transmissão entre a engrenagem motora e a movida, sendo utilizada neste experimento a engrenagem com 24 dentes no eixo motor e variações na engrenagem do eixo movido, sendo utilizadas as engrenagens com 15, 24 e 32 dentes (Figura 3).

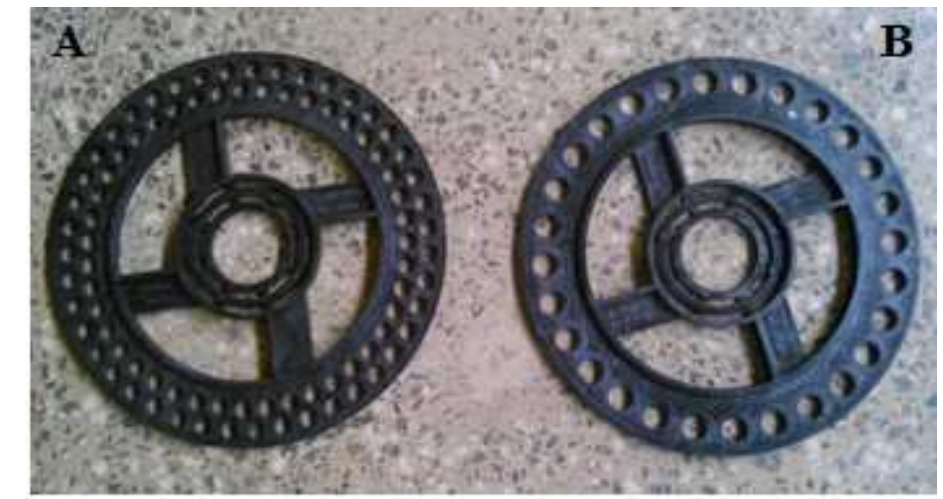

FIGURA 2 - Discos horizontais com formato Redondo com fileira dupla (A), e simples (B), UFCA, Crato, 2016.

Fonte: Autores

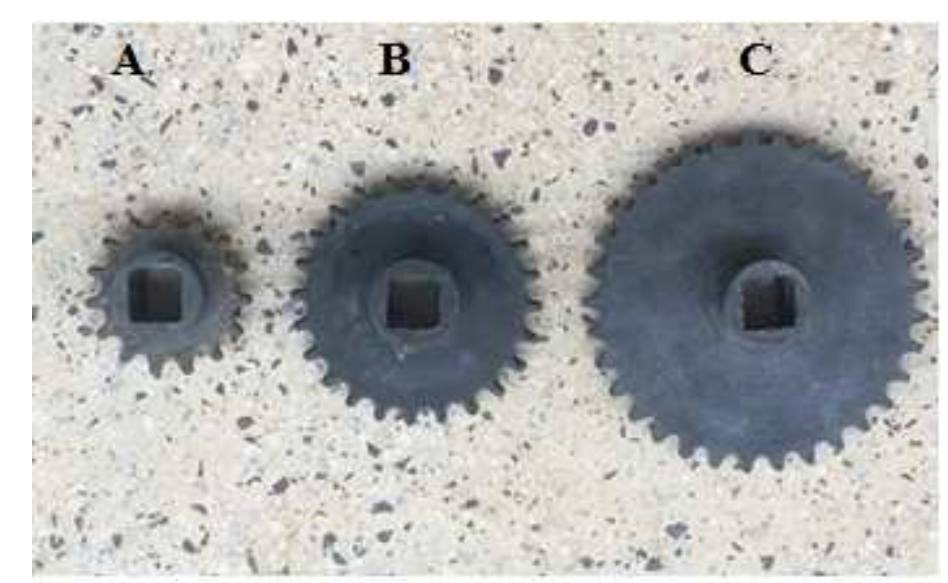

FIGURA 3 - Engrenagens utilizadas no eixo movido de 15 dentes (A), 24 dentes (B) e 32 dentes (C), UFCA, Crato, 2016.

Fonte: Autores

As variáveis analisadas foram a altura de plantas $(\mathrm{cm})$, número de plantas por metro na fileira e por metro quadrado, a massa por planta ( $\mathrm{g}$ ), a massa de plantas por metro de fileira $(\mathrm{g})$ e a produtividade de coentro $\left(\mathrm{g} \mathrm{m}^{-2}\right)$. Após a colheita das plantas contidas na área útil da parcela $\left(1,2 \mathrm{~m}^{2}\right)$, foi mensurado a altura de 20 plantas coletadas ao acaso, dentre as colhidas para análise de produtividade, medindo-se com régua graduada o comprimento da base da planta até o ápice.

Em seguida foi quantificado o número de plantas contido em cada parcela útil, sendo obtidos os valores de plantas por metro de fileira e por metro quadrado. As 
plantas foram pesadas em balança semianalitica, com precisão de duas casas decimais para gramas, com os resultados sendo transformados para massa de plantas por metro de fileira e produtividade de massa fresca.Todos os dados foram submetidos à análise de variância e ao teste de comparação de médias de Tukey a $5 \%$ de probabilidade, utilizando-se o software estatístico SISVAR 5.3 (FERREIRA, 2011).

\section{RESULTADOS E DISCUSSÃO}

Não houve interação entre o tipo de disco e o número de dentes da engrenagem movida para todas as variáveis em estudo (Tabelas 1 e 2). Ao analisar individualmente cada fator, verifica-se na tabela 1, que para a altura de planta 0 disco com fileira dupla não se diferenciou do disco de fileira simples, no entanto quanto ao número de dentes, o disco com 15 dentes apresentou o melhor resultado, isso por que, quanto menor o número de dentes na engrenagem movida maior é a frequência de rotação do disco, consequentemente ocorrerá uma maior deposição de sementes, acarretando em maior competição por luz entre as plantas e maior altura, fato também observado por LIMA et al. (2007).

TABELA 1. Síntese da análise de variância e do teste de médias para a altura da plantas (Altura), o número de plantas por metro (Plantas $/ m$ ) e número de plantas por metro quadrado (Plantas $/ \mathrm{m}^{2}$ ), UFCA, Crato-CE, 2016.

\begin{tabular}{|c|c|c|c|}
\hline \multirow{2}{*}{ Fontes de Variação } & \multicolumn{3}{|c|}{ Quadrados Médios } \\
\hline & Altura & Plantas/m & Plantas $/ \mathrm{m}^{2}$ \\
\hline DISCO (D) & $1,55^{\mathrm{NS}}$ & 1093 ** & $12149 * *$ \\
\hline NÚMERO DE DENTES (N) & $10,41^{N S}$ & 1492 ** & 16579 ** \\
\hline $\mathrm{D} \times \mathrm{N}$ & $0,88^{N S}$ & $141^{\text {NS }}$ & $1572^{\text {NS }}$ \\
\hline erro & 2,74 & 64 & 717 \\
\hline CV\% & 9,20 & 16,78 & 16,77 \\
\hline Teste de Médias & $\begin{array}{c}\text { Altura } \\
\mathrm{cm}\end{array}$ & Plantas/m & Plantas/m² \\
\hline \multicolumn{4}{|l|}{ DISCO (D) } \\
\hline D1 - Fileira Dupla & 18,26 a & $54,7 \mathrm{a}$ & $182,2 \mathrm{a}$ \\
\hline D2 - Fileira Simples & $17,75 \mathrm{a}$ & $41,2 b$ & $137,2 \mathrm{~b}$ \\
\hline \multicolumn{4}{|l|}{ NÚMERO DE DENTES (N) } \\
\hline N1 - 15 Dentes & $19,24 \mathrm{a}$ & $58,5 \mathrm{a}$ & $195,0 \mathrm{a}$ \\
\hline N2 - 24 Dentes & $17,79 a b$ & $52,7 \mathrm{a}$ & $175,8 \mathrm{a}$ \\
\hline N3 - 32 Dentes & $16,99 \mathrm{~b}$ & $32,5 \mathrm{~b}$ & $108,3 \mathrm{~b}$ \\
\hline
\end{tabular}

Já com relação ao número de plantas por metro e o número de plantas por metros quadrados foi significativo tanto com relação ao modelo de disco utilizado 
quanto ao número de dentes. Sendo que o disco com fileira dupla apresentou o melhor resultado. Isso pode ser justificado por que o disco com fileira dupla apesar do diâmetro de cada orifício ser menor ele apresenta o dobro de furos, fato que possibilita um fluxo de deposição contínua de sementes, enquanto que no disco de fileira simples a deposição das sementes pode apresentar intervalos na sua distribuição.

$\mathrm{Na}$ tabela 2 a massa por planta não apresentou diferença significativa entre os modelos de discos e nem quanto ao número de dentes da engrenagem movida.

A Massa de planta por metro e a produtividade foram estatisticamente significativas tanto com relação ao modelo de disco quanto ao número de dentes, sendo que o disco com fileira dupla e com 15 dentes na engrenagem movida demostraram os melhores resultados. Para GRANJEIRO et al. (2011), o coentro é uma cultura que se adapta bem em cultivos com alta densidade de plantas, tanto em cultivo solteiro como em consórcio sem prejudicar a produtividade da mesma, fato que justifica a maior produtividade para o disco com fileira dupla e para a engrenagem com 15 dentes, que apresentaram também a maior população de plantas.

TABELA 2. Síntese da análise de variância e do teste de médias para a massa de coentro por planta (massa/Planta), a massa de plantas por metro (MassaPlantas $/ \mathrm{m}$ ) e a produtividade de coentro (Produtividade), UFCA, Crato-CE, 2016.

\begin{tabular}{|c|c|c|c|}
\hline \multirow{2}{*}{ Fontes de Variação } & \multicolumn{3}{|c|}{ Quadrados Médios } \\
\hline & Massa/Planta & MassaPlantas/m & Produtividade \\
\hline DISCO (D) & $0,36^{\mathrm{NS}}$ & $4187^{* *}$ & 46519 ** \\
\hline $\begin{array}{l}\text { NÚMERO DE DENTES } \\
(\mathrm{N})\end{array}$ & $0,54^{N S}$ & 8785 ** & $97615^{* *}$ \\
\hline $\mathrm{D} \times \mathrm{N}$ & $0,17^{\mathrm{NS}}$ & $1295^{N S}$ & $14390^{N S}$ \\
\hline erro & 0,38 & 377 & 4192 \\
\hline CV\% & 21,89 & 14,81 & 14,81 \\
\hline Teste de Médias & Massa/Planta & MassaPlantas/m & $\begin{array}{l}\text { Produtividade } \\
\qquad \mathrm{g} \mathrm{m}^{-2}\end{array}$ \\
\hline \multicolumn{4}{|l|}{ DISCO (D) } \\
\hline D1 - Fileira Dupla & $2,72 \mathrm{a}$ & $144,3 \mathrm{a}$ & $481,1 \mathrm{a}$ \\
\hline D2 - Fileira Simples & $2,97 \mathrm{a}$ & $117,9 \mathrm{~b}$ & $393,0 \mathrm{~b}$ \\
\hline
\end{tabular}

NÚMERO DE DENTES

$(\mathrm{N})$

$\begin{array}{llcl}\text { N1 }-15 \text { Dentes } & 2,80 \mathrm{a} & 162,4 \mathrm{a} & 541,2 \mathrm{a} \\ \text { N2 }-24 \text { Dentes } & 2,61 \mathrm{a} & 134,6 \mathrm{~b} & 448,7 \mathrm{~b} \\ \text { N3 }-32 \text { Dentes } & 3,13 \mathrm{a} & 96,4 \mathrm{c} & 321,2 \mathrm{c}\end{array}$

Médias seguidas pela mesma letra minúscula na coluna, não diferem entre si pelo teste de Tukey a $5 \%$ de probabilidade.

${ }^{\star *}$ : significativo $(P<0,01)$; *: significativo $(P<0,05)$; NS: não significativo; CV\%: coeficiente de variação. 
SOUSA et al. (2011) ao estudarem o tratamento pré germinativo e densidade de semeadura do coentro em canteiros na Paraíba, observaram que ao utilizarem uma densidade de planta de $3,0 \mathrm{~g} \mathrm{~m}^{-1}$ obtiveram o máximo de produtividade $(1800 \mathrm{~g}$ $\mathrm{m}^{-2}$ ) com uma média de 800 plantas por metros quadrados, tais resultados são superiores aos encontrados neste trabalho, evidenciando a necessidade de novos estudos com discos maiores e outras engrenagens motoras e movidas para elevar a densidade populacional de plantas.

\section{CONCLUSÃO}

Conclui-se que a maior produtividade e número de plantas do coentro verdão foi com o disco de fileira dupla e para a engrenagem movida com 15 dentes.

\section{REFERÊNCIAS}

ANGELI, K. P; DELAZARI, F. T.; NICK, C.; FERREIRA; M. G.; \& DERLY J. H. DA SILVA, D J. H. Yield components and water use efficiency in coriander under irrigation and nitrogen fertilization. Revista Brasileira Engenharia Agrícola e Ambiental, v.20, n.5, p.415-420, 2016. Disponível em: http://dx.doi.org/10.1590/1807-1929/agriambi.v20n5p415-420.

EMBRAPA, Empresa Brasileira de pesquisa Agropecuária, Sistema Brasileiro de Classificação de Solos. 2013. Disponível em: < http://livraria.sct.embrapa.br/liv_resumos/pdf/00053080.pdf> Acessado em 21 de setembro de 2016.

FERREIRA, D. F. Sisvar: A computer statistical analyssis system. Revista Ciência Agrotecnologia, Lavras, v. 35, n. 6, p. 1039-1042, 2011. Disponível em: http://dx.doi.org/10.1590/S1413-70542011000600001

FRANCETTO, T. R.; ALONÇO, A. DOS S.; BELLÉ, M. P.; FRANCK, C. J.; CARPES, D. P. Comportamento operacional de associações entre sulcadores e discos de corte para sistema de semeadura direta. Revista Brasileira Engenharia Agrícola e Ambiental, Jaboticabal, v.35, n.3, p.542-554, 2015. Disponível em: http://dx.doi.org/10.1590/1809-4430-Eng.Agric.v35n3p542-554/2015.

GRANGEIRO, L. C.; SANTOS, A. P.; FREITAS, F. C. L.; SIMÃO, L. M. C.; NETO, F. B. Avaliação agroeconômica das culturas da beterraba e coentro em função da época de estabelecimento do consórcio1 Revista Ciência Agronômica, v. 42, n. 1, p. 242-248, 2011. Disponível em: http://dx.doi.org/10.1590/S180666902011000100030 .

HORTIVALE. Hortivale - Sementes do Vale Ltda. 2015. Disponível em: http://www.hortivale.com.br/. Acesso em: 01 de outubro de 2016.

ILVA, M.A.D.; COELHO JÚNIOR, L.F.; SANTOS, A.P. Vigor de sementes de coentro (Coriandrum sativum L.) provenientes de sistemas orgânicos e convencional Revista Brasileira Plantas Medicinais, Botucatu, v.14, n.esp., p.192-196, 2012. Disponível em: http://dx.doi.org/10.1590/S1516-05722012000500012. 
KÖEPPEN, W. Climatologia: con um estúdio de los climas de la Tierra. México: Fondo de Cultura Econômica, 1948. 478p.

LINHARES, P. C. F.; PEREIRA, M.F.S.; MOREIRA, J.C.1; PAIVA, A.C.C.2; ASSIS, J.P.; SOUSA, R.P Rendimento do coentro (Coriandrum sativum $L$ ) adubado com esterco bovino em diferentes doses e tempos de incorporação no solo. Revista Brasileira Plantas Medicinais, Campinas, v.17, n.3, p.462-467, 2015. Disponível em: http://dx.doi.org/10.1590/1983-084X/13_006.

LIMA; J.S.S., NETO, F. B., NEGREIROS, M. Z., FREITAS, K. K. C., JÚNIOR, A. P. B. Desempenho agroeconômico de coentro em função de espaçamentos e em dois cultivos. Revista Ciência Agronômica, v.38, n.4, p.407-413, 2007. Disponível em: http://dx.doi.org/10.1590/S1806-66902013000100012.

MACIEL GM; COSTA CP; SALA FC. Linhagens de coentro com pendoamento tardio sob dois sistemas de plantio. Horticultura Brasileira, v. 30 n. 1, p. 607-612, 2012. Disponível em: http://dx.doi.org/10.1590/S0102-05362012000400008.

MELO, R. P.; ALBIERO, D.; MONTEIRO, L. A.; SOUZA, F. H.; SILVA, J. G.; Qualidade na distribuição de sementes de milho em semeadoras em um solo cearense. Revista Ciência Agronômica, v. 44, n. 1, p. 94-101, 2013. Disponível em: http://dx.doi.org/10.1590/S1806-66902013000100012.

RESENDE, A. L. S.; FERREIRA, R. B.; SOUZA, B. Atratividade de adultos de Chrysoperla externa (Hagen, 1861) aos compostos voláteis de coentro, endro e erva-doce (Apiaceae) em condições de laboratório. Revista Ceres, Viçosa, v. 62, n.1, p. 037- 043, 2015. Disponível em: http://dx.doi.org/10.1590/0034737X201562010005.

SOUSA, T.V.; ALKIMIM, E.R.A; DAVID, A.M.S.S.; SÁ, J.R.; PEREIRA, G.A.; AMARO, H.T.R.; MOTA, W.F. Época de colheita e qualidade fisiológica de sementes de coentro produzidas no Norte de Minas Gerais Revista Brasileira Plantas Medicinais, Botucatu, v.13, especial, p.591-597, 2011. http://dx.doi.org/10.1590/S1516-05722011000500015.

TAVELLA, L. B.; GALVÃO, R. O.; FERREIRA, R. L. F.; NETO, S. E. A.; NEGREIROS, J. R. S. Cultivo orgânico de coentro em plantio direto utilizando cobertura viva e morta adubado com composto. Revista Ciência Agronômica, v. 41, n. 4, p. 614-618, 2010. http://dx.doi.org/10.1590/S1806-66902010000400014. 